\title{
Design of a Switched Capacitor Negative Feedback Circuit for a Very Low Level DC Current Amplifier
}

\author{
Hiroki Higa, Naoki Nakamura \\ Faculty of Engineering, University of the Ryukyus, Okinawa, Japan \\ Email: hrhiga@eve.u-ryukyu.ac.jp
}

Received May 2, 2013; revised June 2, 2013; accepted June 9, 2013

Copyright (C) 2013 Hiroki Higa, Naoki Nakamura. This is an open access article distributed under the Creative Commons Attribution License, which permits unrestricted use, distribution, and reproduction in any medium, provided the original work is properly cited.

\begin{abstract}
To miniaturize a very low level dc current amplifier and to improve its output response speed, the switched capacitor negative feedback circuit (SCNF), instead of the conventionally used high-ohmage resistor, is presented in this paper. In our system, a switched capacitor filter (SCF) and an offset controller are also used to decrease vibrations and offset voltage at the output of the amplifier using SCNF. The theoretical output voltage of the very low level dc current amplifier using SCNF is obtained. The experimental results show that the unnecessary components of the amplifier's output are much decreased, and that the response speed of the amplifier with both the SCNF and SCF is faster than that using high-ohmage resistor.
\end{abstract}

Keywords: DC Amplifier; Small Current Measurement; Switched Capacitor

\section{Introduction}

Response speeds of the measuring instruments are limited by those of very low level dc current amplifiers [1,2], when very small currents are measured by mass spectroscopes and radiation detectors. This implies that the amplifiers are required to observe rapid transient phenomena. The very low level dc current amplifier for measuring small currents generally consists of an amplifier having high input impedance and a high-ohmage negative feedback resistor. The amplifier with high-ohmage resistor has unavoidable effects of the stray capacitances across its terminals. This factor causes the amplifier to have a complicated frequency characteristic, which results in poor responses of the very low level dc current amplifier [1-3]. Some shielding techniques [4-6] have been reported for the purpose of decreasing these capacitive components. In spite of the fact that these methods have been employed, it was difficult to realize drastic improvements of the response speeds of the very low level dc current amplifier. Neither are the amplifiers with shielding methods appropriate for miniaturization. A positive feedback circuit [7] had also been used as another approach to decrease the stray capacitances. The amplifier however was unstable and began to oscillate in this case. The resultant high speed response of the amplifier has not been achieved so far.

To explore the optimum solution to the above prob- lems, the switched capacitor negative feedback circuit (SCNF) has been developed. The switched capacitor (SC) circuit is equivalent to a resistor and is suitable for miniaturization. A theoretical output voltage of the very low level dc current amplifier using SCNF with a switched capacitor filter (SCF) was investigated in this paper. Furthermore, the output voltage of the amplifier was experimentally demonstrated.

\section{Circuit Description}

Figure 1 summarizes a very low level dc current amplifier, including SCF and a small current source. $C_{g}, R_{g}$ and $K$ are the input capacitance, input resistance, and amplification factor of the amplifier having a high input resistance, respectively. In the experiment, we utilized a triangular wave voltage produced by the function generator $V_{g}$ and the differentiating capacitor $C_{s}$ (reactance attenuator) to obtain a square wave current $I_{s}$ with a high output impedance as an input signal to the amplifier. $C_{o}$ is the output capacitance to the ground of $C_{s}$.

The SCNF and SCF are shown in Figure 2. The SCNF is composed of several analog switches $\left(S_{1}, S_{2}, \cdots, S_{7}\right)$ and capacitors. These switches are controlled by two nonoverlapping clock signals. $S_{1}$ is synchronous with $S_{2}$ and $S_{3}$, and $S_{4}$ is synchronous with $S_{5}, S_{6}$ and $S_{7}$, respectively. An output voltage of the very low level dc current amplifier has vibrations due to charge and discharge actions of 


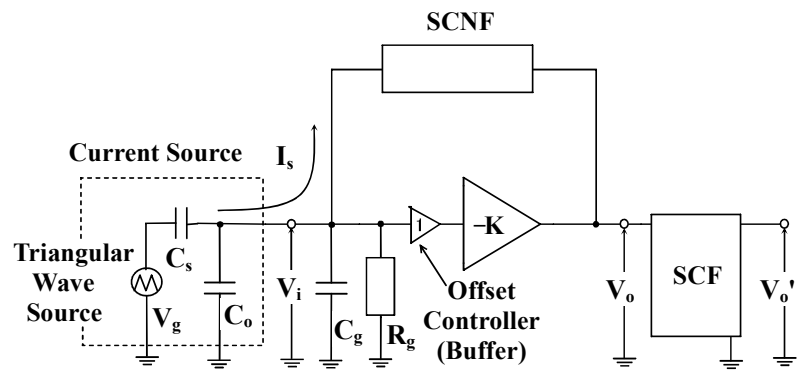

Figure 1. Circuit configuration of very low level de current amplifier. SCNF and SCF stand for switched capacitor negative feedback circuit and switched capacitor filter, respectively.

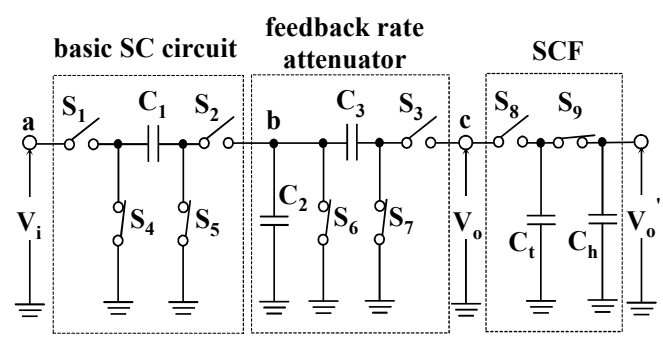

Figure 2. SCNF and SCF.

the SC circuit. Thus, as shown in Figure 2, the SCF was connected to the output of the amplifier. The switches $S_{8}$ and $S_{9}$ are synchronous with $S_{1}$ and $S_{4}$, respectively.

\section{Circuit Analysis}

This section describes an equivalent resistance of SCNF and theoretical output voltage of the very low level dc current amplifier. In the following analysis, it is assumed that $T_{1}$ is the time when $S_{1}, S_{2}$, and $S_{3}$ are closed $\left(S_{4}, S_{5}\right.$, $S_{6}$, and $S_{7}$ are opened), and $T_{2}$ is the time when $S_{1}, S_{2}$, and $S_{3}$ are opened $\left(S_{4}, S_{5}, S_{6}\right.$, and $S_{7}$ are closed). Further, it is assumed that a small current $I_{s}$ is dc current because the clock frequency of the SC circuit, $f_{s}$, is much higher than the frequency of $I_{s}$.

\subsection{Equivalent Resistance of SCNF}

From Figure 2, the voltage at node $b, V_{b}$, is represented by

$$
V_{b}=\frac{C_{3}}{C_{2}+C_{3}} V_{o},
$$

and an electric charge $q_{1}$ at $C_{1}$ is

$$
q_{1}=C_{1}\left(V_{b}-V_{i}\right) \text {. }
$$

From Equation (1) and the relationship that $V_{o}=-K V_{i}$, the electric charge $q_{1}$ at $C_{1}$ can be rewritten as

$$
q_{1}=C_{1}\left(\frac{C_{3}}{C_{2}+C_{3}} V_{o}+\frac{V_{o}}{K}\right) \approx C_{1} V_{o} \frac{C_{2}+K C_{3}}{K\left(C_{2}+C_{3}\right)},
$$

for $K \gg 1$. Because the electric charge $q_{1}$ at $C_{1}$ during $T_{2}$ is totally discharged, the quantity of the charge that is transported from node $a$ to node $b$ is equivalent to $q_{1}$. Thus, a current, $I$, flowing from node $a$ into node $b$ during one clock cycle $T_{s}$ is

$$
I=\frac{q_{1}}{T_{s}}=V_{o} \frac{C_{1}}{T_{s}} \cdot \frac{C_{2}+K C_{3}}{K\left(C_{2}+C_{3}\right)} .
$$

Since the current to be measured in the very low level dc current amplifier $I_{s}$ flows into the SC circuit, $I_{s}=I$. From the relationship that $V_{o}=R_{f e q} I_{s}$ [1], the equivalent resistance of SCNF $R_{f e q}$ is given by

$$
R_{f e q}=\frac{T_{s}}{C_{1}} \cdot \frac{K\left(C_{2}+C_{3}\right)}{C_{2}+K C_{3}},
$$

while the equivalent resistance of the $\mathrm{SC}$ circuit [8] $R_{s c}$ is represented by

$$
R_{s c}=\frac{T_{s}}{C_{1}}=\frac{1}{C_{1} f_{s}},
$$

where $f_{s}$ is the clock frequency. The attenuation factor of the attenuator $X[9]$ becomes

$$
X=\frac{C_{2}+K C_{3}}{K\left(C_{2}+C_{3}\right)} .
$$

Hence, from Equations (4) to (6), $R_{f e q}$ can be obtained as

$$
R_{f e q}=\frac{1}{X C_{1} f_{s}}=\frac{R_{s c}}{X} .
$$

From Equation (6), it is observed that $X$ is dependent on the ratio of capacitances of $C_{2}$ and $C_{3}$.

\subsection{Theoretical Output Voltage of the Amplifier}

Output voltage of the very low level dc current amplifier using SCNF has vibrations due to charge and discharge actions of the SC circuit. In this section, theoretical output voltage of the very low level dc current amplifier is discussed.

The equivalent circuit of the SC circuit is shown in Figure 3(a). It is found from Equations (5) and (7) that the SCNF is equivalent to the capacitor of $X C_{1}$ and four switches $S_{1}, S_{3}, S_{4}$, and $S_{7}$. The equivalent circuit of the very low level dc current amplifier is shown in Figure 3(b). The box labeled "*” in Figure 3(b) stands for the SCNF and this figure shows that the equivalent circuit of the SCNF shown in Figure 3(a) is connected with the equivalent circuit of the amplifier at the terminals between nodes $a$ and $c$.

Millman's theorem is applied to Figure 3(b), and the voltage $V_{i}$ is represented by

$$
V_{i}=\frac{I_{s}+j \omega X C_{1}\left(-K V_{i}\right)}{1 / R_{g}+j \omega\left(C_{o}+C_{g}+X C_{1}\right)},
$$




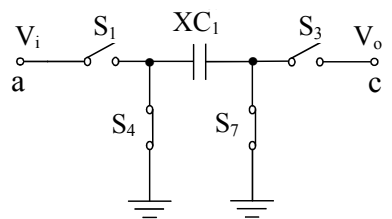

(a)

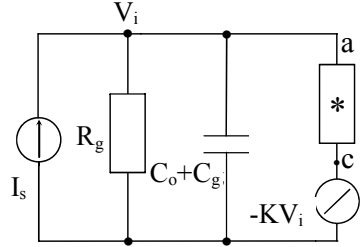

(b)

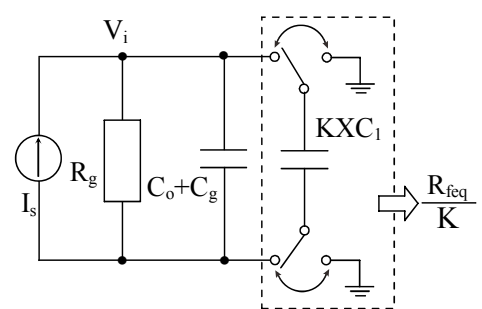

(c)

Figure 3. Equivalent circuits of (a) SCNF and (b) very low level de current amplifier. Figure 3(c) shows simplified input equivalent circuit of Figure 3(b).

where $\omega$ is the angular frequency. The input admittance of the very low level dc current amplifier $Y_{\text {in }}$ is

$$
Y_{i n}=\frac{I_{s}}{V_{i}} \approx \frac{1}{R_{g}}+j \omega\left(C_{o}+C_{g}+K X C_{1}\right),
$$

for $K \gg 1$. Using Equation (8), a simplified input equivalent circuit of the very low level dc current amplifier using SCNF can be drawn as shown in Figure 3(c).

An enlarged input voltage waveform of the amplifier at the positive final steady-state, $V_{i}$, is illustrated with the help of clock waveforms as shown in Figure 4. $T_{s}$ is the clock cycle of the switches. $T_{1}$ and $T_{2}$ are $(1-\alpha) T_{s}$ and $\alpha T_{s}$, respectively. Let the input voltage of the amplifier at $t=n T_{s}$ be $V_{i}(n)$. Subscript symbols "+" and "-" mean just after and just before the time event occurs, respectively. For example, $V_{o}(n+1)$ - means the voltage just before $t=(n+1) T_{s}$. The amplitude of the input voltage for a cycle, $V_{m}$, is

$$
\begin{aligned}
V_{m}= & \frac{1}{C_{o}+C_{g}+K X C_{1}} \int_{n T_{s}}^{(n+(1-\alpha)) T_{s}} I_{s} \mathrm{~d} t \\
& +\frac{1}{C_{o}+C_{g}} \int_{(n+(1-\alpha)) T_{s}}^{(n+1) T_{s}} I_{s} \mathrm{~d} t \\
= & \frac{C_{o}+C_{g}+\alpha K X C_{1}}{\left(C_{o}+C_{g}\right)\left(C_{o}+C_{g}+K X C_{1}\right)} I_{s} T_{s} .
\end{aligned}
$$

Since electric charges of the SC circuit are conserved just before and after $t=n T_{s}$, the following equation is obtained

$$
\left(C_{o}+C_{g}\right) V_{i}(n)_{-}=\left(C_{o}+C_{g}+K X C_{1}\right) V_{i}(n)_{+} .
$$

The input voltage just before $t=n T_{s}$ is

$$
V_{i}(n)_{-}=\left(1+\frac{K X C_{1}}{C_{o}+C_{g}}\right) V_{i}(n)_{+} .
$$

From Figure 4 and Equation (10), the voltage $V_{m}$ is

$$
V_{m}=V_{i}(n)_{-}-V_{i}(n)_{+}=\frac{K X C_{1}}{C_{o}+C_{g}} V_{i}(n)_{+} .
$$

From Equations (9) and (11), the input voltage just after $t$ $=n T_{s}$ can be obtained as

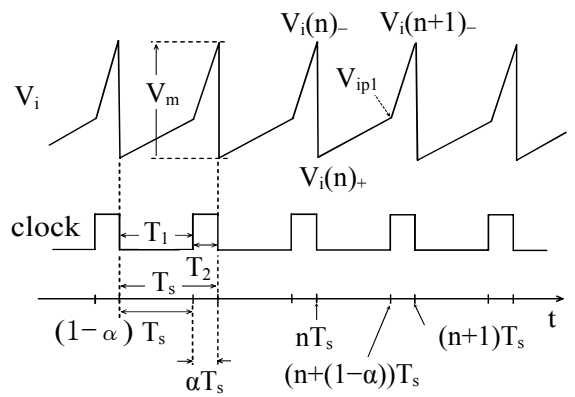

Figure 4. Relationship between enlarged input voltage and clock waveforms.

$$
V_{i}(n)_{+}=\frac{I_{s} T_{s}}{K X C_{1}} \cdot \frac{C_{o}+C_{g}+\alpha K X C_{1}}{C_{o}+C_{g}+K X C_{1}} .
$$

Thus the peak voltage $T_{i p 1}$ during $T_{1}$ is

$$
\begin{aligned}
V_{i p 1} & =V_{i}(n)_{+}+\frac{1}{C_{o}+C_{g}+K X C_{1}} \int_{n T_{s}}^{(n+(1-\alpha)) T_{s}} I_{s} \mathrm{~d} t \\
V_{i p 1} & =V_{i}(n)_{+}+\frac{1}{C_{o}+C_{g}+K X C_{1}} \int_{n T_{s}}^{(n+(1-\alpha)) T_{s}} I_{s} \mathrm{~d} t \\
& =V_{i}(n)_{+}+\frac{1-\alpha}{C_{o}+C_{g}+K X C_{1}} I_{s} T_{s} .
\end{aligned}
$$

Substituting Equation (12) into Equation (13) gives the following equation:

$$
V_{i p 1}=\frac{I_{s} T_{s}}{K X C_{1}}=I_{s} \frac{R_{f e q}}{K} .
$$

Therefore, the peak output voltage of the amplifier during $T_{1}, V_{o p 1}$, can be written as

$$
V_{o p 1}=-K V_{i p 1}=-I_{s} R_{f e q} .
$$

It is seen from Equation (15) that the theoretical output voltage of the very low level dc current amplifier using SCNF can be obtained by sampling $V_{o p 1}$, and that the equivalent resistance, $R_{f e q}$, is independent of duty ratio of the clock signal.

In this paper, the SCF was used to sample $V_{o p 1}$ from the output voltage of the very low level dc current amplifier using SCNF for the following reasons. Using a sample-and-hold circuit generally requires a clock generator 
that completely differs from two non-overlapping clock signals utilized by the SCNF, and using a low-pass filter provides for not theoretical output voltage, but approximately half amplitude of output voltage of the amplifier at a final steady-state. On the other hand, using the SCF allows for sharing the two non-overlapping clock signals. Both the SCF and SCNF can be also manufacturable by the same process. Therefore, the SCF is useful from the viewpoint of miniaturization.

\section{Simulation Results}

Using the electronic circuit simulator PSpice (Cadence Design System, Inc.), transient analyses of the very low level dc current amplifier using SCNF were carried out. $K$ and $C_{g}$ were set to 1300 and $17 \mathrm{pF}$, respectively. The equivalent resistance of the SC circuit $R_{R C}$ was set to 1 $\mathrm{M} \Omega$ by using $C_{1}$ of $10 \mathrm{pF}$ and $f_{s}$ of $100 \mathrm{kHz}$. The attenuation factor $X$ of $1 / 100$ was also set by using both $C_{2}$ of $1000 \mathrm{pF}$ and $C_{3}$ of $9.3 \mathrm{pF}$. Thus, the total equivalent resistance $R_{f e q}$ of the SCNF was $100 \mathrm{M} \Omega$. The duty ratio of the clock cycle, $\alpha$, was also set to 0.5 . To evaluate response speeds of the very low level dc current amplifier, a square wave current $I_{s}$ with a time period of $5 \mathrm{~ms}$ and an amplitude of $10 \mathrm{nA}$ was input to the amplifier. From Equation (15), output voltage of $1 \mathrm{~V}$ should be obtained as the theoretical output voltage of the amplifier. A switch model [10] used in the computer simulation is shown in Figure 5. The symbols G, D, and S stand for gate, drain and source of a MOS-FET. Each analog switch is composed of a combination of an nMOS and pMOS, as shown in Figure 5(a). Assuming that parasitic capacitances between two terminals exist, as shown in
Figures 5(b) and (c), the transient analyses by computer simulation were conducted.

First, it is assumed that nMOS has exactly the same parasitic capacitances as pMOS has. The parasitic capacitance values are shown in Table 1. These values were determined by trial and error. Figures 6(a) and (b) show the simulation results in the case of using the parasitic capacitances shown in Table 1. It can be seen that the output voltage of the amplifier has vibrations due to charge and discharge actions of the SCNF, and that the vibrations of output of the amplifier causes black areas in the output waveform (see Figures 6(a) and (b)).Thus, it is difficult to measure an input current from the output waveform of the amplifier. Average values of $V_{o p 1}$ from $10 \mathrm{~ms}$ to $12.5 \mathrm{~ms}$ and from $12.5 \mathrm{~ms}$ to $15 \mathrm{~ms}$ in Figure 6(a) were obtained as $+1.0 \mathrm{~V}$ and $-1.0 \mathrm{~V}$, respectively. The simulation result in the case of using the SCF at the output of the amplifier is shown in Figure 6(c). In this case, the peaks of the output voltage during $T_{1}$ were sampled by the SCF. The rise time of the output waveform of the SCF is $20.0 \mu \mathrm{s}$. As defined in general, the rise time is the time required for the output waveform to rise from $10 \%$ to $90 \%$ of its final steady-state value. It is clear from Figure 6(c) that using the SCF drastically reduces vibrations as well as unnecessary components, and that the input current $I_{s}$ can be obtained by measuring the amplitude of its output voltage.

Secondly, on the assumption that nMOS and pMOS have totally different parasitic capacitances, as shown in Table 2, computer simulations of transient analyses of the very low-level dc current amplifier using SCNF were also performed. The parasitic capacitance values indicated

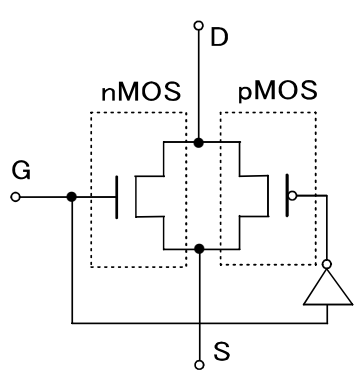

(a)

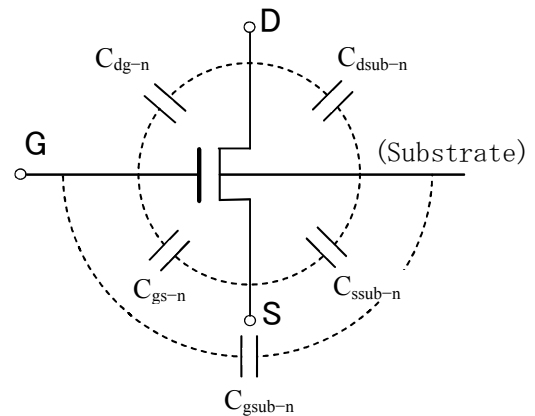

(b)

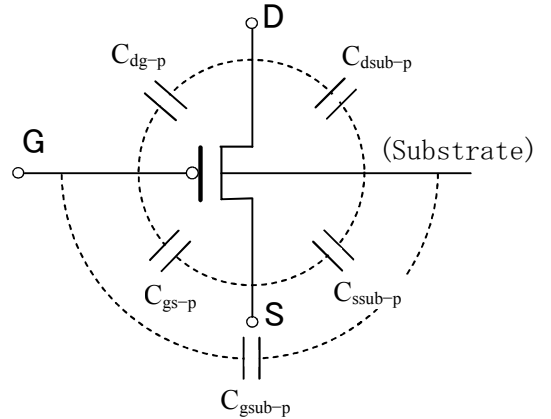

(c)

Figure 5. Switch model used in PSpice simulation. (a) Configuration of CMOS switch, (b) nMOS and (c) pMOS switch models with parasitic capacitances.

Table 1. Values of parasitic capacitances in the case of assumption that nMOS and pMOS have the same parasitic capacitive components.

\begin{tabular}{ccccc}
\hline \multicolumn{5}{c}{ parasitic capacitance [pF] } \\
\hline$C_{d g-n}, C_{d g-p}$ & $C_{g s-n}, C_{g s-p}$ & $C_{d s u b-n}, C_{d s u b-p}$ & $C_{s s u b-n}, C_{s s u b-p}$ & $C_{g s u b-n}, C_{g s u b-p}$ \\
0.10 & 0.09 & 0.07 & 0.07 & 0.06 \\
\hline
\end{tabular}




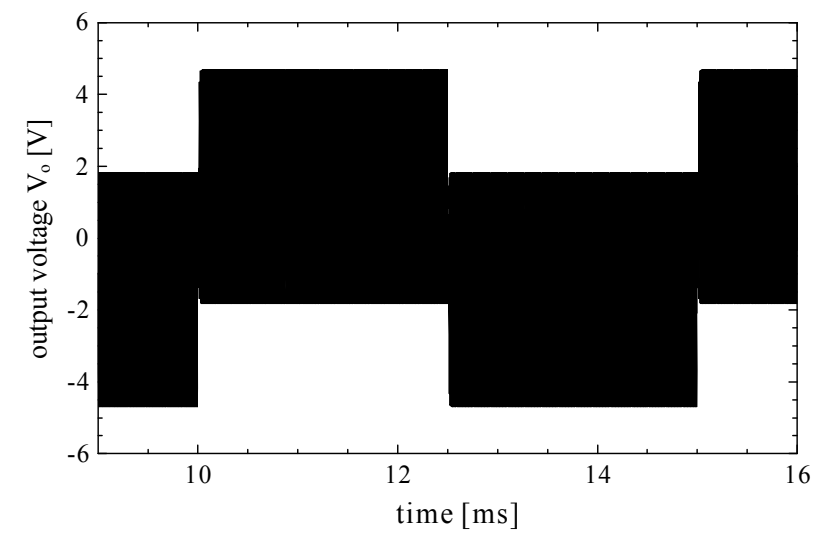

(a)

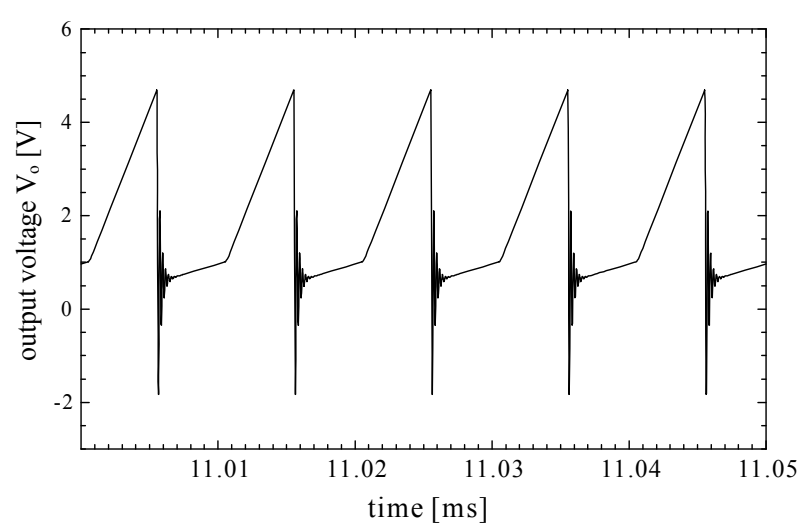

(b)

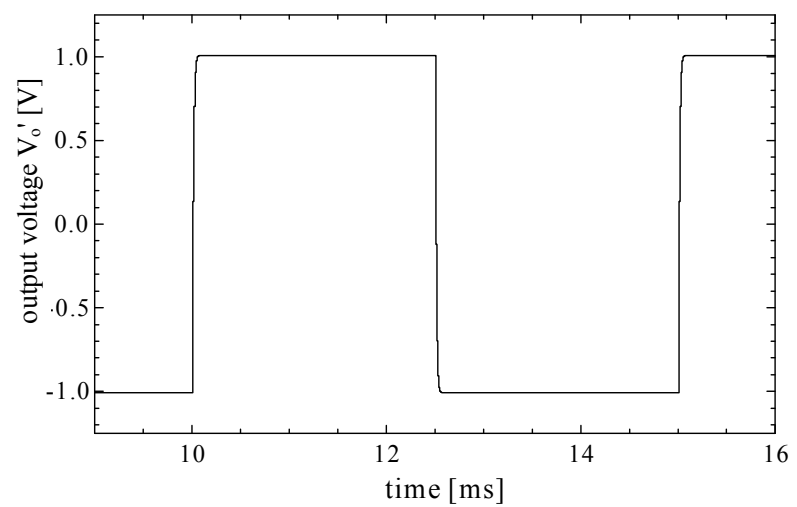

(c)

Figure 6. Simulation results in the case of using parasitic capacitances shown in Table 1. (a) Output waveform of very low level dc current amplifier using SCNF, (b) its enlarged waveform at a positive final steady-state, and (c) output waveform of $\mathrm{SCF}$. The rise time of output waveform in Figure 6(c) is $20.0 \mu \mathrm{s}$.

Table 2. Values of parasitic capacitances in the case of assumption that nMOS and pMOS have totally different parasitic capacitive components.

\begin{tabular}{cccccc}
\hline \multicolumn{5}{c}{ parasitic capacitance $[\mathrm{pF}]$} \\
\hline$S_{1}$ & $C_{d g-n}$ & $C_{g s-n}$ & $C_{\text {dsub-n }}$ & $C_{\text {ssub-n }}$ & $C_{\text {gsub-n }}$ \\
$S_{2}$ & 0.08 & 0.13 & 0.06 & 0.05 & 0.05 \\
$S_{3}$ & 0.09 & 0.10 & 0.09 & 0.05 & 0.06 \\
$S_{4}$ & 0.07 & 0.14 & 0.08 & 0.06 & 0.06 \\
$S_{5}$ & 0.07 & 0.11 & 0.07 & 0.09 & 0.06 \\
$S_{6}$ & 0.10 & 0.13 & 0.08 & 0.07 & 0.06 \\
$S_{7}$ & 0.10 & 0.14 & 0.07 & 0.07 & 0.06 \\
& $C_{d g-p}$ & $C_{g s-p}$ & $C_{d s u b-p}$ & $C_{\text {ssub-p }}$ & $C_{\text {gsub-p }}$ \\
$S_{1}$ & 0.15 & 0.09 & 0.07 & 0.07 & 0.06 \\
$S_{2}$ & 0.11 & 0.07 & 0.06 & 0.06 & 0.08 \\
$S_{3}$ & 0.12 & 0.09 & 0.06 & 0.07 & 0.06 \\
$S_{4}$ & 0.15 & 0.06 & 0.06 & 0.07 & 0.06 \\
$S_{5}$ & 0.14 & 0.12 & 0.10 & 0.08 & 0.09 \\
$S_{6}$ & 0.14 & 0.09 & 0.04 & 0.05 & 0.06 \\
$S_{7}$ & 0.14 & 0.08 & 0.10 & 0.09 & 0.08 \\
\hline
\end{tabular}

in Table 2 were also determined by trial and error. Figures 7(a) and (b) show the simulation results in the case of using the parasitic capacitances shown in Table 2 . It can be found from Figure 7(a) that the output voltage of the amplifier has also vibrations, as shown in Figure 6(a), and that the amplitudes of the black painted areas are roughly 5 times larger than those in Figure 6(a). Further, it is seen that the output waveform of the amplifier has rapid changes in output voltage from $T_{1}$ to $T_{2}$, and that the rapid change $V_{c}$ is $-25.7 \mathrm{~V}$ (see Figure 7(b)). The simulation result in the case of using the SCF at the output of the amplifier is shown in Figure 7(c). The rise time of the output waveform of the SCF is also $20.0 \mu \mathrm{s}$. It is obvious from Figure 7(c) that the amplitude of the output voltage of the amplifier becomes $1 \mathrm{~V}$, and that the output waveform has the offset voltage of $2.04 \mathrm{~V}$. Comparing Figure 7 with Figure 6, it is thought that a clock feed through generated by totally different parasitic capacitive components leads to a generation of the offset and the rapid changes in output voltage of the very low level dc current amplifier using SCNF. From other simulation results, it was found that using different parasitic capacitances of $C_{d g-n}$ and $C_{d g-p}$, and those of $C_{g s-n}$ and 


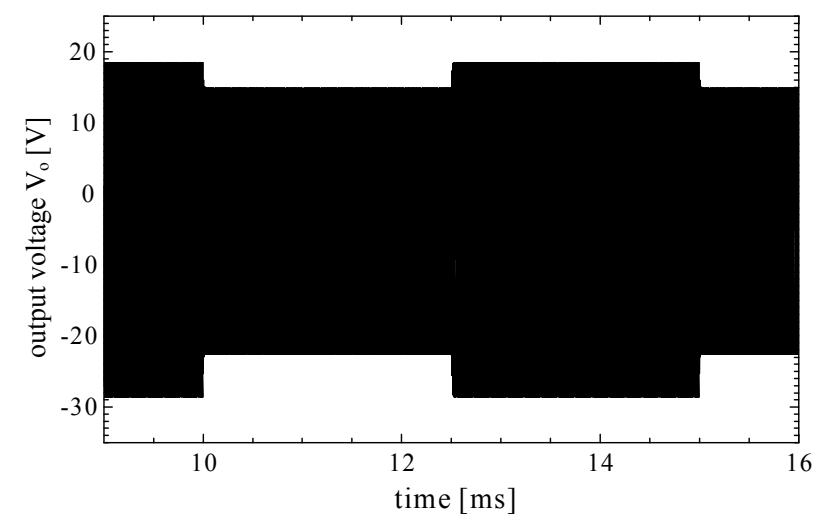

(a)

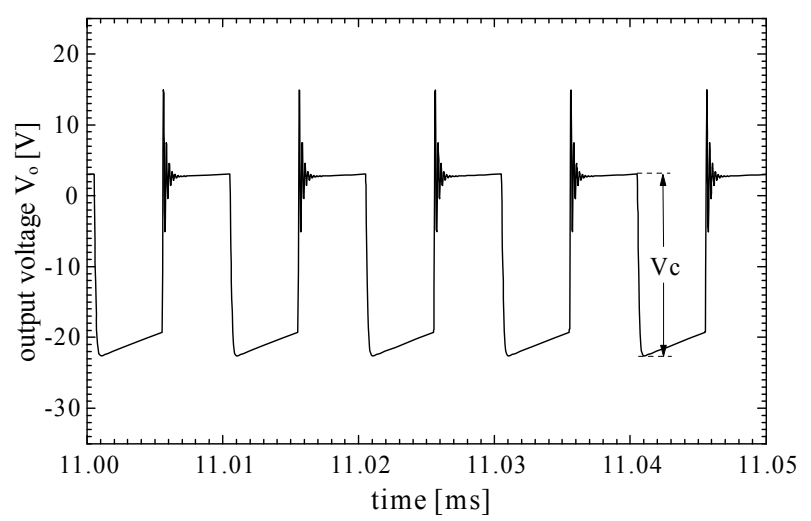

(b)

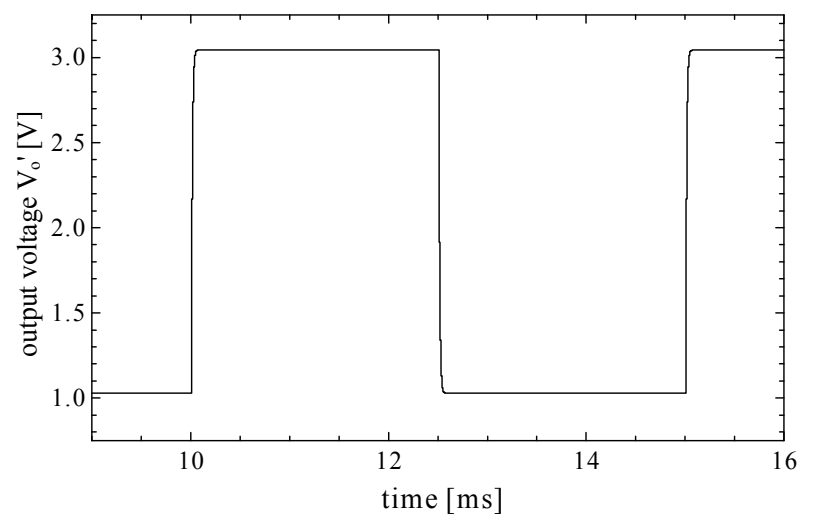

(c)

Figure 7. Simulation results in the case of using parasitic capacitances shown in Table 2. (a) Output waveform of very low level dc current amplifier using SCNF, (b) its enlarged waveform at a positive final steady-state, and (c) output waveform of $\mathrm{SCF}$. The rise time of output waveform in Figure 7(c) is $\mathbf{2 0 . 0} \mu \mathrm{s}$.

$C_{g s-p}$ resulted in the generation of offset voltage of the amplifier, and that using larger parasitic capacitances of $C_{d s u b-n}, C_{d s u b-p}, C_{s s u b-n}$ and $C_{s s u b-p}$ led to an error of the equivalent resistance $R_{\text {feq }}$. On the other hand, parasitic capacitances of $C_{g s u b-n}$ and $C_{g s u b-p}$ did not have an effect on the output voltage of the amplifier.

\section{Experimental Results and Discussion}

First, the very low level dc current amplifier using SCNF shown in Figure 1 was made and its output responses were observed. The amplifier having high input resistance was composed of two differential amplification stages and an emitter follower stage, and the first stage had two JFETs to obtain high input resistance of the amplifier. The amplification factor $K$ of the amplifier was $62 \mathrm{~dB}$ (From DC to $1.2 \mathrm{MHz}$ ), and output voltage waveform was observed using an oscilloscope. Since the triangular wave voltage, which had a time period of $5 \mathrm{~ms}$ and an amplitude of $10 \mathrm{~V}$, was differentiated by the differentiating capacitor $C_{s}$ of $1.25 \mathrm{pF}$, a square wave current with a time period of $5 \mathrm{~ms}$ and an amplitude of 10 nA was obtained as an input current $I_{s}$ to the amplifier. As switches of the SC circuit, we used CMOS analog switches (MAX326, MAXIM Integrated Products, Inc.) having the maximum leakage current of $10 \mathrm{pA}$. Further, variable capacitors $C_{1}$ and $C_{3}$ were utilized. Parasitic capacitances of analog switches have a little effect on equivalent resistance of the SCNF $R_{\text {feq }}$, which causes errors in $R_{f e q}$ of the amplifier. Thus, the equivalent resistance $R_{s c}$ of the SC circuit with the clock frequency $f_{s}$ of $100 \mathrm{kHz}$, was set to $1 \mathrm{M} \Omega$ by adjusting capacitance of $C_{1}$, and then the attenuation factor of the feedback rate attenuator $X$ was set to $1 / 100$ by adjusting capacitance of $C_{3}$. Referring to Equation (7), the total equivalent resistance of the SCNF became $100 \mathrm{M} \Omega$. An offset voltage controller, which was connected to the input of the amplifier and had a gain of unity, was also used to cancel the offset voltage in the experiment. The input stage of the offset controller was composed of a JFET which had much higher input impedance than the negative feedbackcircuit had, and its voltage drift was very small (several $\mu \mathrm{V}$ ). Therefore, the offset controller did not have much effect on the current detection sensitivity of the amplifier.

Figures 8(a) and (b) show the output voltage waveform of the very low level dc current amplifier using 


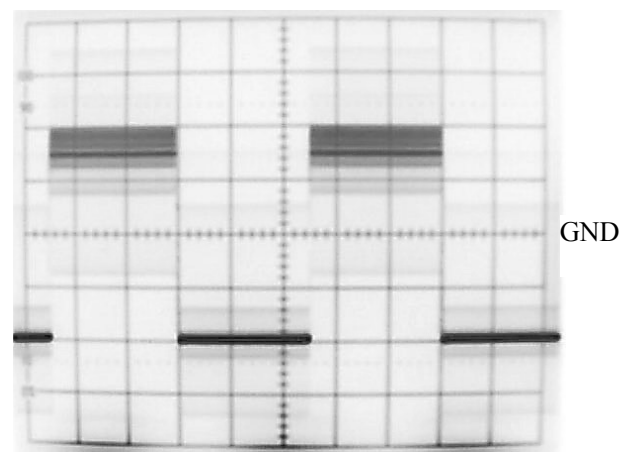

(a)

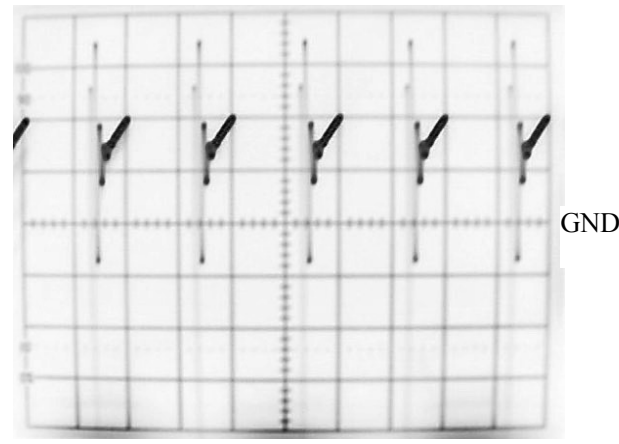

(b)

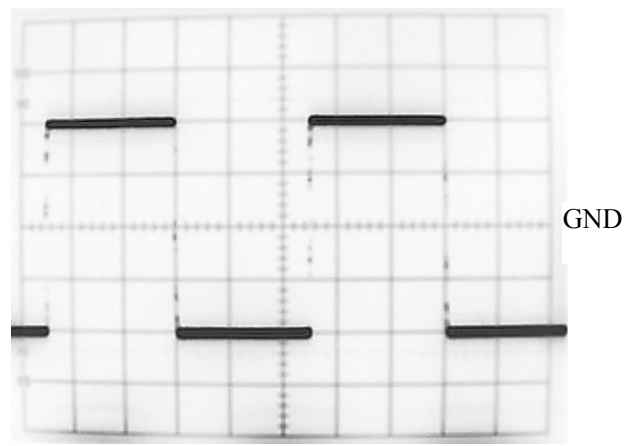

(c)

Figure 8. Experimental results. (a) Output waveform (scale H: $1 \mathrm{~ms} / \mathrm{div}, \mathrm{V}: 0.5 \mathrm{~V} / \mathrm{div}$ ), (b) its enlarged waveform (scale H: $5 \mu \mathrm{s} / \mathrm{div}, \mathrm{V}: 0.5 \mathrm{~V} / \mathrm{div}$ ) of very low level de current amplifier using SCNF, and (c) output waveform (scale $H$ : 1 $\mathrm{ms} / \mathrm{div}, \mathrm{V}: 0.5 \mathrm{~V} / \mathrm{div}$ ) of SCF, consisting of $C_{t}$ of $470 \mathrm{pF}$ and $C_{h}$ of $220 \mathrm{pF}$. The rise time of output waveform in Figure 8 (c) is $23.8 \mu \mathrm{s}$.

SCNF and its enlarged waveform at a positive final steady-state, respectively. It is seen from Figure 8(a) that the experimental output waveform does not extensively have the black area as shown in Figure 6(a) and 7(a). This is because rapid changes in output voltage of the amplifier do not appear on a CRT display of the oscilloscope due to its resolution, and as a matter of fact vibrations due to charge and discharge actions of the SCNF can be found at any enlarged waveforms in Figure 8(b). From this figure, it is clear that the output waveform of the amplifier has vibrations and rapid changes as ob- served in Figure 7(b). Because the amplifier in the PSpice simulation does have an amplitude limiter, in the case of simulation result in Figure 7(b), the minimum output voltage of the amplifier during $T_{2}$ was indicated $-22.6 \mathrm{~V}$, while in the case of experimental result, the output voltage of the amplifier during $T_{2}$ was saturated at $-13 \mathrm{~V}$ due to the power supply of the negative voltage of the amplifier.

Peak values of the output voltage during $T_{1}$ were sampled by the SCF. The experimental result is shown in Figure 8(c). It is clear from Figure 8(c) that using the SCF drastically reduces vibrations of the output of the amplifier, and that the theoretical input current $I_{s}$ can be obtained by measuring the amplitude value of its output voltage. Thus it is possible to measure an input current of the very low level dc current amplifier using SCNF with the SCF. With respect to the Figure 8(c), the rise time of the output waveform of the SCF is $23.8 \mu \mathrm{s}$. On the other hand, that of the output waveform of the very low level dc current amplifier using conventionally used high-ohmage resistor is $92.0 \mu \mathrm{s}$. We are easily available to miniaturize SC circuits using IC-compatible techniques. Therefore, using the SCNF is an effective way to obtain both a faster output response and miniaturization of a very low level dc current amplifier.

Finally, a relationship between the equivalent resistance of the SCNF $R_{f e q}$ and the clock frequency $f_{s}$ was investigated. It is clear from Equation (7) that $R_{f e q}$ can be set using the clock frequency and capacitances of $C_{1}, C_{2}$, and $C_{3}$. In this experiment, we first set to $f_{s}$ of $100 \mathrm{kHz}$ and $C_{2}$ of $1000 \mathrm{pF}$, adjusted both capacitances of $C_{1}$ and $C_{3}$ to precisely obtain $R_{f e q}$ of $100 \mathrm{M} \Omega$, and then changed $f_{s}$ ranging from $50 \mathrm{kHz}$ to $200 \mathrm{kHz}$. The equivalent resistance of SCNF was obtained by measuring output voltage of the SCF. The relationship between $R_{f e q}$ and $f_{s}$ and error rate of $R_{\text {feq }}$, in the case of $X$ of $1 / 100$, are shown in Figure 9. Theoretical values of $R_{f e q}$ in Figure 9(a) were calculated using Equation (7) with $C_{1}$ of $10.0 \mathrm{pF}, C_{2}$ of $1000 \mathrm{pF}$, and $C_{3}$ of $9.32 \mathrm{pF}$. It is obvious from Figures 9(a) and (b) that the experimental curve agrees well with the theoretical one, and that the error rate of $R_{f e q}$ is within $0.86 \%$ for the theoretical values. From this experimental result, it is thought that $R_{\text {feq }}$ ranging from $1.1 \mathrm{M} \Omega$ with $X$ of $9 / 10$ to $1.0 \mathrm{G} \Omega$ with $X$ of $1 / 500$ will be settable.

\section{Conclusion}

A theoretical output voltage of a very low level de current amplifier using SCNF with SCF was derived from circuit analysis. It was experimentally demonstrated that a small current of $10 \mathrm{nA}$ could be measured using the amplifier, and that response speed of the amplifier using SCNF was faster than that of the amplifier using conventionally used high-ohmage resistor. A consideration of a 


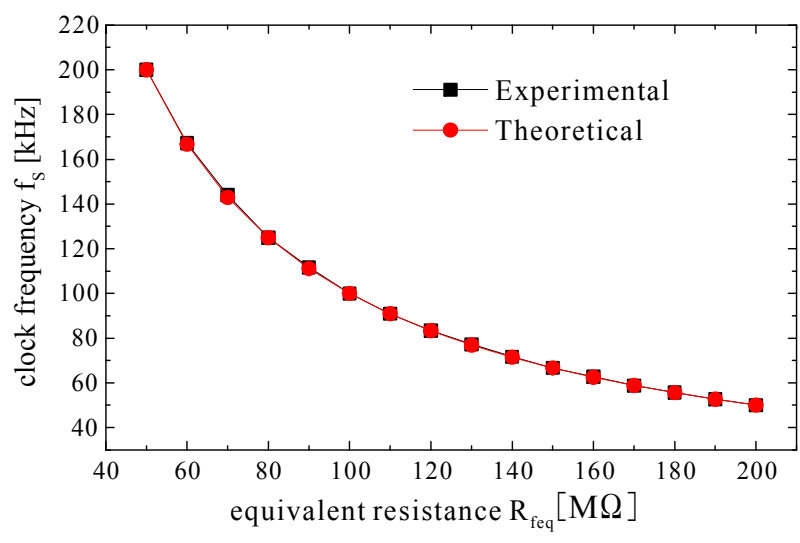

(a)

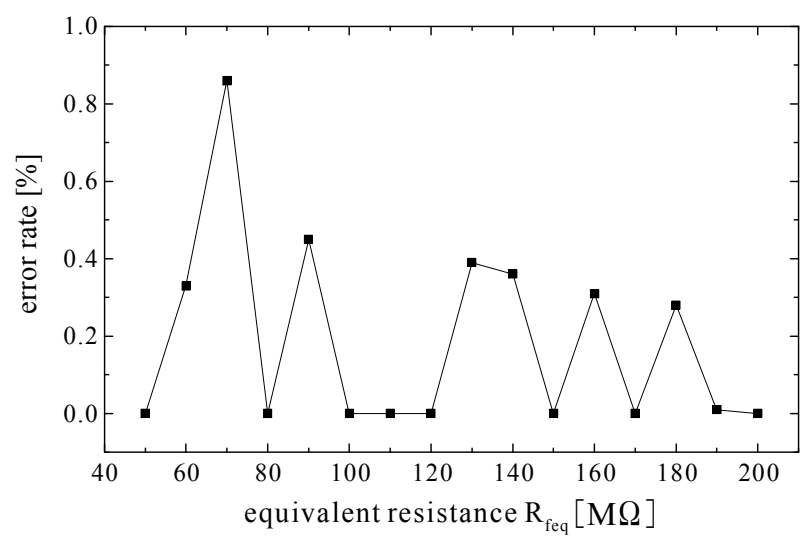

(b)

Figure 9. Experimental result: (a) relationship between $\boldsymbol{R}_{\text {feq }}$ and $f_{s}$ and (b) error rate of $\boldsymbol{R}_{f e q}$.

very low level dc current amplifier using SCNF with SCF will be needed for practical applications as our future work.

\section{REFERENCES}

[1] S. P. Presley, "Fast Response Picoammeter," Review of
Scientific Instruments, Vol. 37, No. 5, 1966, pp. 643-648. doi:10.1063/1.1720272

[2] K. Goto and K. Ishikawa, "Design and Construction of High Speed Pico-Ammeter," The Journal of the Vacuum Society of Japan, Vol. 22, No. 6, 1979, pp. 235-246. doi:10.3131/jvsj.22.235

[3] I. Nakamura and T. Kano, "Noise and Fast Response of a Very Small dc Current Amplifier," Oyo Buturi, Vol. 52, No. 4, 1983, pp. 330-338.

[4] F. Galliana and P. P. Capra, "Hamon $10 \times 100 \mathrm{M} \Omega \mathrm{Re}-$ sistor Based Traceable Source for Calibration of Picoammeters in the Range 100 pA -100 nA," Measurement, Vol. 43, No. 9, 2010, pp. 1277-1281. doi:10.1016/i.measurement.2010.07.006

[5] F. Galliana, P. P. Capra and E. Gasparotto, "Metrological Management of the High dc Resistance Scale at INRIM," Measurement, Vol. 42, No. 2, 2009, pp. 314-321. doi:10.1016/j.measurement.2008.07.002

[6] I. Nakamura and M. Takemura, "Fast Respoinse of a Very Low-Level dc Current Amplifier Using Improvement of Feedback Resistor Shielding Structure," IEICE Transactions on Electronics, Vol. J72-C-II, No. 10, 1989, pp. 885-892.

[7] I. Nakamura and T. Kano, "High Speed Response of a Very Low Level dc Current Amplifier Using Positive Feedback Loop," Oyo Buturi, Vol. 54, No. 9, 1985, pp. 945-951.

[8] A. B. Grebene, "Bipolar and MOS Analog Integrated Circuit Design,” John Wiley \& Sons Inc., Hoboken, 1984.

[9] H. Higa, R. Onaga, N. Nakamura and I. Nakamura, "A Basic Study on a Very Low-Level dc Current Amplifier Using a Switched-Capacitor Circuit: Comparison between Simulation and Experimental Results," Proceedings of ITC-CSCC2005, Jeju, 7 July 2005, pp. 1133-1134.

[10] N. H. E. Weste and K. Eshraghian "Principle of CMOS VLSI Design: A Systems Perspective,” T. Tomisawa and Y. Matsuyama, Translation, Maruzen, 1999. 